\title{
Kongressbericht: 2. COMBATing-Kongress 13.-14.11.2009 "Vom molekularen Phantasialand zur zielgerichteten Realität»
}

\author{
Julia Herold Rachel Wuerstlein Marwa Salem \\ Klinik und Poliklinik für Frauenheilkunde und Geburtshilfe, Uniklinik Köln, Germany
}

Der 2. COMBATing-Kongress (Conference On Molecular Basics And Therapeutic implications in breast cancer) wurde im Phantasialand in Brühl bei Köln von Frau Prof. Harbeck (Köln) eröffnet, die zusammen mit Herrn Prof. Kaufmann (Frankfurt/M.) und Herrn PD Dr. Rody (Frankfurt/M.) die wissenschaftliche Leitung hatte. Sie begrüßte die 200 Teilnehmer aus unterschiedlichsten Bereichen (Klinik, Forschung, Pharmaindustrie, Studierende), die hauptsächlich aus Österreich, der Schweiz und Deutschland kamen.

Prof. Emons (Göttingen), Vorsitzender der Arbeitsgemeinschaft Gynäkologische Onkologie (AGO), stellte in seinem Grußwort die jüngste Kommission der AGO vor: die Kommission für Translationale Forschung (TraFo). Er bedauerte die noch zu geringe Unterstützung der Kommission TraFo von Seiten der Pharmaindustrie, die eher an der Entwicklung molekularer Prozesse interessiert sei als an der praktischen Umsetzung in der Klinik. Er betonte weiterhin die Relevanz der internationalen und interdisziplinären Zusammenarbeit. Dabei unterstrich er die Sonderstellung des Mammakarzinoms (MC) in Deutschland als Aufgabengebiet der Gynäkologen. Dr. Bruns (Berlin), Generalsekretär der Deutschen Krebsgesellschaft, betonte in seiner Grußbotschaft, dass Translationalität genau so wichtig sei wie Interdisziplinarität.

Die erste Vortragsreihe «Update - Was gibt es Neues seit Combating 2008?» leiteten Prof. Jakesz (Wien), PD Dr. Müller (Hamburg) und Prof. Niederacher (Düsseldorf). Prof. Sinn (Heidelberg) berichtete über die «Hot Topics der Tumorentstehung». Er hob die B3-Klassifikation im Screening hervor, die kein Synonym für eine Vorläuferläsion, sondern eine prozedurale Klassifikation sei, die das diagnostische Management beeinflussen sollte. Seinen Schwerpunkt setzte er auf die erste neoplastische Vorstufe FEA (flache epitheliale Atypie) als duktale Vorläufer-Läsion des Zylinderepithels. Die FEA ist somit der Beginn der Karzionogenese des invasiv-duktalen MC. Die Grenzziehung zwischen benigner und maligner Lä- sion wird hiermit von ADH (atypische duktale Hyperplasie) auf die FEA vorverlagert.

Prof. Meindl (München), Koordinator Analytik des Konsortiums Erbliches Mamma- und Ovarialkarzinom, berichtete über das hereditäre MC «beyond BRCA». Seine Arbeitsgruppe hat kürzlich eine neue Spleiß-Mutation eines Tumorsuppressorgens identifiziert, die zirka 3\% der nicht-BRCA induzierten familiären $\mathrm{MC}$ erklärt; erste Ergebnisse dieser Arbeit werden demnächst im JCO publiziert.

Herr Gluz (Mönchengladbach) referierte über das Thema «Therapieresistenz: Wissen wir heute mehr?». Dabei legte er den Schwerpunkt auf das umliegende Stromagewebe als Prädiktor der Entwicklung einer Chemoresistenz (stromal metagen score, smc). Neue zielgerichtete Strategien wie z.B. mTOR-Inhibitoren sind mögliche Ansatzpunkte zur Überwindung der Chemotherapieresistenz. Derzeit gibt es jedoch keine Marker oder Signaturen, die außerhalb von klinischen Studien Anwendung finden dürfen.

Prof. Schneeweiß (Heidelberg) referierte über die Vor- und Nachteile der antiangiogenen Therapie beim MC und verglich die verschiedenen Strategien zur Inhibition des Vascular Endothelial Growth Factors (VEGF): Die Small Molecules wie z.B. Sunitinb und Sorafenib als Tyrosinkinase-Inhibitoren, die neutralisierenden Antikörper (z.B. Bevazcizumab), lösliche Rezeptoren wie VEGF-Traps (Ligandenfänger) und die Hemmung der VEGF-Produktion (antisense/siRNA).

Durch die zweite Vortragsreihe mit dem Thema «Neue Entwicklungen in der Translationalen Forschung» führten Frau Prof. Fehm (Tübingen), Prof. Huober (St. Gallen) und Prof. Maass (Aachen). Frau Dr. Hannemann (Hamburg) präsentierte eine Zusammenfassung der prognostischen und prädiktiven Bedeutung der Multigenanalysen. Insbesondere die kommerziell erhältlichen Signaturen MammaPrint und Oncotype DX finden zur Unterstützung der Therapieentscheidung zunehmend Anwendung. Das Problem der Intermediären

\section{KARGER}

Fax +497614520714

Information@Karger.de

www.karger.com
(C) 2009 S. Karger GmbH, Freiburg

Accessible online at:

www.karger.com/brc
Julia Herold

Uniklinik Köln

Klinik und Poliklinik für Frauenheilkunde und Geburtshilfe

Kerpener Str. 34, 50931 Köln, Germany

Tel. +49 221 478-4959, Fax +49 221 478-97439

julia.herold@uk-koeln.de 
Gruppe konnte bis dato jedoch nicht zufriedenstellend gelöst werden. Hierzu werden die Auswertungen der aktuell laufenden Studien (z.B. TAILORx und Plan B) zur Evaluation der prognostischen und prädiktiven Relevanz der Multigenanalysen mit Spannung erwartet.

Prof. Thomssen (Halle) ging in seinem Vortrag auf die Problematik des G2-Gradings ein, das auf 70\% der Hormonrezeptor-positiven (HR+) MC zutrifft. Eine Entscheidungshilfe bietet hier die uPA/PAI-1-Testung, die durch die Einfachheit und Stabilität bei geringen Kosten überzeugt. Der Nachteil ist die zwingend notwendige Asservierung von Frischgewebe des Tumors. Im Gegensatz zu den Multigenassays (Oncotype DX, MammaPrint) gibt es hier prospektive Daten. Dabei ist die Validität der uPA/PAI-1-Testung so evident, dass sie sich bereits in den AGO-Leitlinien etabliert hat.

Frau Dr. Rack (München) beschrieb die minimale Resterkrankung (minimal residual disease, MRD) als möglichen Ansatzpunkt der antitumorösen Therapie. Die Persistenz isolierter Tumorzellen im Knochenmark sowie zirkulierende Tumorzellen im Blut sind von prognostischer Relevanz sowohl zum Zeitpunkt der Primärdiagnose als auch in der Nachsorge. Die MRD-Diagnostik kann der Selektion von Risikopatientinnen in der rezidivfreien Nachsorge, die möglicherweise von einer zusätzlichen Therapie profitieren, sowie dem Therapiemonitoring in der (neo)adjuvanten und metastasierten Situation dienen. MRD kann als therapeutische Zielstruktur für zellzyklusunabhängige Therapieinterventionen, wie z.B. die Bisphophonat-Therapie, angegangen werden.

Die Trägerin des diesjährigen COMBATing-Awards, Dr. Liedtke (Münster), präsentierte die Problematik des triplenegativen MC: Ein Tumor ohne Target? Die Zielsetzung bei Patientinnen aus diesem Risikokollektiv sei zum einen die Identifizierung der Patientinnen, die auf eine Therapie ansprechen, zum anderen die Optimierung herkömmlicher Chemotherapien. Ein weiteres Ziel ist die Entwicklung bzw. Nutzung neuer Therapien, wie beispielsweise antiangiogene Substanzen, sowohl intra- als auch extrazellulär.

Den Abschluss des ersten Kongresstages bildete die Round-Table-Diskussion mit Vertretern aus der Klinik, der Forschung, der Industrie, der Politik und einer Vertreterin der Patienten. Durch die Diskussion «Translationale Forschung: gefordert, aber nicht gefördert: medizinische, gesundheitsökonomische und patientenbezogene Erwartungen und Widersprüche» führte TV-Moderator und Autor Thomas Hegemann (Heidelberg) (Abb. 1). Prof. Wiesler vom Deutschen Krebsforschungszentrum Heidelberg betonte die Wichtigkeit der Verbindung zwischen Grundlagenforschung und Klinik. Durch Zentren wie die Comprehensive Cancer Centers (CCC) sei es möglich, beides unter einem Dach zu vereinigen. Dabei müssten den Klinikern Freiräume für Forschung geschaffen werden. Er verwies dabei auf die Position des «Physician Scientist», der als Kliniker in die Forschung integriert wird mit Strukturen, die die Vereinbarkeit mit der alläglichen Arbeit in der Klinik möglich machen. Auch Prof.

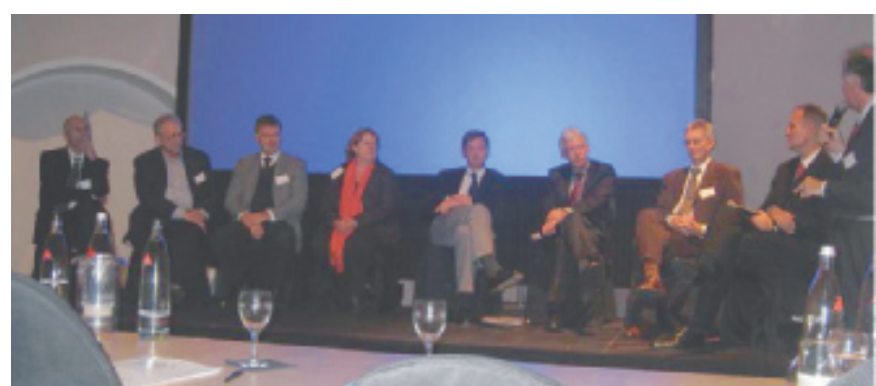

Abb. 1. Die Teilnehmer der Round-Table-Diskussion. Von links nach rechts: PD Dr. Rody, Prof. Schmidt, Dr. Bruns, Frau Haidinger, Prof. Wiesler, Herr Nettekoven, Herr Ameismeier, Prof. Schneeweiß, Moderator Thomas Hegemann.

Schmitt, Leiter der klinischen Forschergruppe der Frauenklinik und Poliklinik der TU München, betonte die Relevanz der Arbeit von Klinikern in der Forschung. Dabei forderte er kein Rotationsprinzip, vielmehr sollte die Wissenschaft in die klinische Arbeit integriert werden. Dafür muss der klinisch tätige Arzt im Bereich der molekularen Grundlagen ein fundiertes Wissen besitzen. Nur so sei es möglich, die Erkenntnisse der Forschung zeitnah an den Patienten zu bringen. Prof. Schneeweiß, als Vertreter der Deutschen Gesellschaft für Senologie, unterstrich die bedeutende Rolle der Klinikleitung. Besonders diese stehe in der Verantwortung, motivierten Ärzten die Freiräume zu gewährleisten, die benötigt würden, um die neuesten Entwicklungen in der Forschung «from bench to bedside» und zurück zu bringen. PD Dr. Rody bewertete die abnehmende Anzahl an Habilitationen in Deutschland als besorgniserregend. Die Wissenschaft müsse wieder attraktiv gemacht werden. Er kritisierte, dass die akademische Forschung kein Bestandteil der Weiterbildung und somit nicht in den ärztlichen Alltag integriert sei. Die Bildung von «onkologischen Spitzenzentren» forderte Gerd Nettekoven, Geschäftsführer der Deutschen Krebshilfe e.V. Des Weiteren sollten mehr Anträge zur Förderung der Wissenschaft bei der Deutschen Forschungsgemeinschaft, dem Bundesministerium für Bildung und Forschung sowie der Deutschen Krebshilfe gestellt werden. Dr. Ameismeier von der Sektion $\mathrm{C}$ der Deutschen Krebsgesellschaft bewertete die Pharmaindustrie als «Teamplayer», die der Dynamik der Onkologie Rechnung trage. Im Gegensatz zu den Krankenkassen und der Politik investiere die Industrie in den Forschungsstandpunkt Deutschland. Die Kooperation zwischen Pharmaindustrie und Klinik müsse optimiert werden. Frau Haidinger, Patientenvertreterin, wies darauf hin, dass translationale Forschung für die Patientin nicht fassbar sei. Deshalb sei der Nutzen dieser Art von Wissenschaft für Patienten schwer nachvollziehbar. Dr. Bruns, Deutsche Krebsgesellschaft, sieht die gesetzliche Krankenversicherung in der zentralen Rolle der Koordination.

Die Verleihung der mit jeweils 500€ dotierten KongressStipendien für junge Wissenschaftler bzw. Ärzte erfolgte 


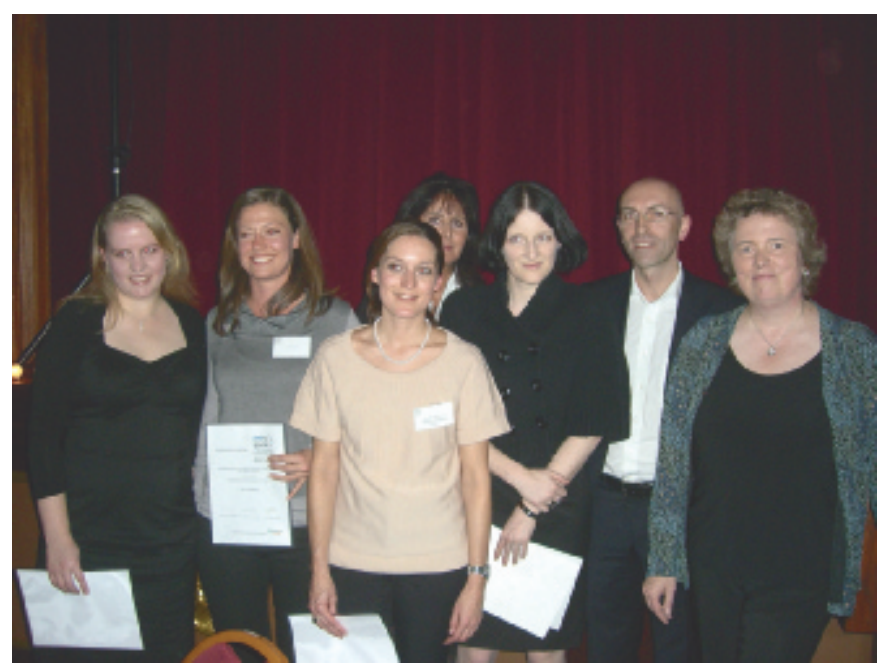

Abb. 2. Verleihung der Kongress-Stipendien und des COMBATingAwards 2009: Von links nach rechts: Kathrina Michels, Julia Herold, Dr. Anna Emde, Sonja Haase, Novartis, Dr. Cornelia Liedtke, PD Dr. Rody, Prof. Harbeck.

durch Frau Prof. Harbeck, PD Dr. Rody und Sonja Haase, Novartis (Abb. 2). Die Preisträger sind Dr. Anna Emde (Israel), Dr. Atanas Ignatov (Magdeburg), Julia Herold (Köln) und Kathrina Michels (Münster). Den COMBATingAward 2009 mit einer Prämie von $2500 €$ erhielt Frau Dr. Cornelia Liedtke (Münster) für herausragende wissenschaftliche Leistungen.

Der folgende Kongresstag begann mit der Key Note Lecture von Dr. Ignatiadis (Brüssel) zum Thema «Incorporation of translational research into clinical trials» mit Schwerpunkt auf der frühzeitigen Identifizierung von biologischen Markern im neoadjuvanten Setting zur Optimierung der individuellen Tumortherapie.

Den Vorsitz in der Vortragsreihe «Das molekulare Verständnis um die Metastasierung» hatten Prof. Jakesz (Wien), Prof. Janni (Düsseldorf) und Prof. Arnold (Kiel). Dr. Schütz (Heidelberg) berichtete über neue therapeutische Möglichkeiten in Hinsicht auf Immunsystem und Tumorzelle. Dass die Immunreaktion auf Tumorzellen bei einigen Tumorpatienten unterdrückt ist, erklärt er mit seinen neuesten Untersuchungen anhand von regulatorische T-Zellen (Tregs), die die tumorspezifische T-Zell-Antwort inhibieren. Als therapeutischer Ansatz ist die Depletion von Tregs und der Einsatz von tumor-reaktiven T-Gedächtniszellen des Knochenmarks möglich. PD Dr. Fasching (Erlangen/Los Angeles) referierte über endokrin resistente Tumore. Er unterschied dabei primäre Resistenz, bei der die Gründe im Wirt oder im Tumor liegen und sekundäre Resistenz, welche sich nach primärem Ansprechen entwickelt. Dass sich präklinische Modelle in die Praxis übertragen lassen, demonstrierte Dr. Fasching anhand diverser Studien und warb für die intensive Mitarbeit an Studien. Prof. Stickeler (Freiburg) beleuchtete Resistenzmechanismen und neue Therapieansätze bei HER2-positivem MC. Die Response nach Trastuzumab-Therapie dauert im Medi- an ein Jahr, wobei zirka 15\% der Patienten nach adjuvanter Trastuzumab-Gabe Metastasen entwickeln. Er erläuterte Resistenzmechanismen gegen Trastuzumab anhand des HERRezeptor-vermittelten Transduktionsweges.

Prof. Schmitt (München) und Dr. Braun (Bonn) hatten den Vorsitz des Themengebiets «Molecular Specials». Frau Dr. Bernhard (Darmstadt) sprach über den adoptiven Transfer von autologen HER2-spezifischen T-Zellklonen bei Patientinnen mit MC. So kann bei Progression nach Chemotherapie und Trastuzumab bei HER2+ Lebermetastasen und disseminierten HER2+ Tumorzellen im Knochenmark durch die Infusion von HER2-spezifischen T-Zellklonen und Interleukin anhand eines Monitorings durch Frequenzanalyse der ${ }^{111}$ Indium-markierten T-Zellen im Blut und Knochenmark das Migrationsverhalten der T-Zellklone nachvollzogen werden. Dr. Beer (München) befasste sich mit der Frage, ob die molekulare Bildgebung wirklich neue Information liefert. Als Beispiel stellte er die quantitative Fluoroestradiol-PET als prädiktive Methode der Response auf die endokrine Therapie bei Brustkrebs vor. Mit dieser Methode kann die Tumorangiogenese sichtbar gemacht werden. Prof. Diel (Mannheim) präsentierte die Pathogenese und Therapie von Knochenmetastasen. Dabei ging er auf die Effekte der Bisphosphonate auf die Tumorzelle ein: Wachstumshemmung, Invasionshemmung, Steigerung der Apoptose sowie reduzierte Häufigkeit von ossären Metastasen und Reduktion der Osteoporose. Die adjuvante Gabe von Bisphosponaten zur Prophylaxe von ossären Metastasen bei prä- und postmenopausalen $\mathrm{Pa}$ tientinnen ist bereits in den AGO-Leitlinien etabliert. Als Alternative zu den Bisphophonaten stellte er die ebenfalls AGO-anerkannten Modulatoren des Receptor Activator of Nuclear Factor Kappa B Liganden (RANKL) vor. RANKL ist der essentielle Mediator, der die Bildung, die Funktion und das Überleben von Osteoklasten bewirkt und der von Osteoblasten exprimiert wird. Denosumab, ein monoklonler Antikörper, hemmt ebenso wie Osteoprotegerin die Bindung des RANKL an RANK, wodurch die Neubildung und Funktion von Osteoklasten und somit die tumorinduzierte Knochendestruktion reduziert wird.

In der letzten Veranstaltungssitzung «Targets and Treatment» waren Frau Prof. Nitz (Mönchengladbach) und PD Dr. Warm (Köln) die Vorsitzenden. Die pharmazeutischen Unternehmen Amgen, GlaxoSmithKline und Novartis stellten ihre «New Kids on the Block» vor: Dr. Hecker von der Firma Amgen präsentierte die biotechnologischen Innovationen der drei großen Einsatzgruppen in der onkologischen Therapie «Targeting Tumor Cells», «Targeting Tumor Stroma» und «Enabling Cancer Care». Er stellte als neuen Ansatz bei Knochenerkrankungen den monoklonalen Antikörper Denosumab vor, der in den Knochenstoffwechsel eingreift. Auf der Ebene des Tumorstromas erläuterte er die Substanz AMG 386: ein Angiopoetin Antagonist Peptibody mit selektiver Inhibition der Interaktion von Angiopoietin 1 und 2 mit dem Tie-2 Rezeptor, einer Rezeptor-Tyrosin- 
Kinase, die auf vaskulären und lymphatischen endothelialen Zellen sowie auf hämatopoetischen Zellen exprimiert wird. Panitumumab (Vectibix ${ }^{\circledR}$ ), der erste vollhumane EGFRAntikörper für die Behandlung von soliden Tumoren, befindet sich derzeit in Phase-II-Studien bei gynäkologischen Tumoren. Auch produziere Amgen innovative Supportiva wie die hoch wirksamen Wachstumsfaktoren der Hämatopoese: PEGylierter humaner Granulozyten-Kolonie stimulierender Faktor (G-CSF) zur Einmalgabe pro Zyklus (Pegfilgrastim), modifiziertes, lang wirksames Erythropoetin (Darbepoetin) sowie den Thrombopoese-stimulierenden Peptibody (Romiplostim).

Dr. Dethling von der Firma GlaxoSmithKline präsentierte den oralen Angiogenesehemmer Pazopanib, der VEGFR-1, $-2,-3$, PDGFR- $\alpha$ und $-\beta$ sowie c-Kit hemmt. Eine randomisierte Phase-II-Studie zeigt, dass eine Therapie mit Pazopanib in Kombination mit Lapatinib vs. Lapatinib mono bei Patienten mit HER2+, fortgeschrittenem MC zu einem verlängerten progressionsfreien Intervall führt (Slamon D et al.: $\mathrm{J}$ Clin Oncol 2008;26:1016 (Meeting Abstracts)). Laut Dethling ist eine weitere Evaluation dieses zytostatikafreien Regimes geplant und stellt ein beeindruckendes neues Paradigma in der Therapie des HER2+ MC dar. Des Weiteren stellte er die Thrombopoetin-Rezeptor-aktivierende Substanz Eltrombopag vor. Diese erhöht für die Dauer der Therapie den Thrombozytenspiegel im Blut, der jedoch nach dem Absetzen rasch abfällt.

Dr. Muth, Medical Advisor von Novartis Oncology, zeigte einen Überblick der «Novartis-Pipeline». Der Multi-Tyrosinkinaseinhibitor TKI 258 besitzt direkte antiproliferative Eigenschaften sowie eine antiangiogene Wirksamkeit. In vitro zeigt sich eine Hemmung der Proliferation bei Mamma- und Ovarial-Karziomen sowie bei anderen Tumoren. Im Gegensatz zu Sunitinib und Sorafenib hemmt TKI 258 zusätzlich Fibroblast Growth Factor Receptor 1 (FGFR1), welcher in 8,5\% der MC überexprimiert wird. Diese Amplifikation geht mit einer signifikant erhöhten Wahrscheinlichkeit für die Entwicklung von Fernmetastasen einher und scheint ein unabhängiger Prädiktor für das Gesamtüberleben zu sein. Für 2010 ist eine Studie im metastasierten Setting geplant. Die Wirksamkeit des oralen mTOR-Inhibitors RAD001 (Everolimus) wird derzeit u. a. in den Studien BOLERO 1-3 sowie in der RADAR-Studie untersucht.

Prof. Möbus (Frankfurt) referierte über die «targeted therapy». Er zeigte Daten zur Wirksamkeit von Pertuzumab und Trastuzumab als initiale Kombinationstherapie und zur Pertuzumab-Therapie nach Progress unter Trastuzumab (Scheuer W et al.: Cancer Res 2009;69(24):OF1-7). Ein signifikant verlängertes Gesamtüberleben im Vergleich zu Trastuzumab mono konnte in beiden Arbeitsgruppen bestätigt werden. T-DM1, an Trastuszumab via Linker gebundene DM1-Chemotherapie, wirkt trotz nachgewiesener PI3K-Mutation oder erniedrigtem PTEN beim HER2+ therapierefraktärem MC. Weiter wurde Neratinib - ein irreversibler, oraler Tyrosinkinase-Inhibitor, der beim HER2+, fortgeschrittenen MC eingesetzt wird vorgestellt. Bei Neratinib-Einnahme mit und ohne vorherige Trastuzumab-Therapie kann sowohl eine erhöhte objektive Ansprechrate als auch eine verlängerte progressionsfreie Zeit verzeichnet werden. Als weitere Innovation stellte Prof. Möbus den PARP-Inhibitor Olaparib vor, der bei BRCATrägerinnen mit fortgeschrittenem, vortherapiertem MC eingesetzt wird. Erste Ergebnisse von Tutt et al. (J Clin Oncol 2009;27:18s (suppl; abstr CRA 501)) zeigen ein erhöhtes Gesamtansprechen. Der IGF-1R-Inhibitor (AMG 479), ein humaner Antikörper, blockiert den IGF-1R, hemmt somit Zellproliferation und begünstigt die Apoptose. Erste präklinische Studien demonstrieren eine Hemmung des Tumorwachstums.

Den abschließenden Vortrag der Veranstaltung hielt Prof. von Minckwitz (Neu-Isenburg) über die aktuellen klinischen Studien u.a. der German Breast Group (GBG). Für das HER2+ MC präsentierte er die Studien ALTTO- und NeoALTTO, GEPARQuinto, BETH, CLEOPATRA und die EMILIA-Studie. Für Patientinnen mit triple-negativem MC eignen sich die Studien BEATRICE und GEPARQuinto, in denen die zusätzliche Gabe von Bevacizumab in der adjuvanten bzw. neoadjuvanten Situation getestet wird. Außerdem wird die Anwendung von PARP-Inhibitoren, wie z.B. BSI-201 in Kombination mit Carboplatin und Gemcitabin beim triple-negativen MC untersucht.

Im Bereich des HR+, HER2-, metastasierten MC gibt es in der First-Line die Studien LEA und TIES. Der mTORInhibitor RAD001 findet in den Studien RADAR und GEPARQuinto Verwendung. In ersterer wird die Monotherapie mit Everolimus untersucht, während in der GEPARQuinto die Addition von Everolimus zu Paclitaxel in der Neoadjuvans analysiert wird. Die ABCSG 18-Studie vergleicht placebo-kontrolliert die zusätzliche Gabe des RANKL-Inhibitors Denosumab mit einem Aromataseinhibitor bei postmenopausalen, rezeptorpositiven Patientinnen.

Auch weitere Studien, die sich noch in Planung befinden, stellte Prof. von Minckwitz vor, darunter auch GEPARSixto, die vor und nach neoadjuvanter Chemotherapie die disseminierten Tumorzellen im Knochenmark und im Blut untersucht. Dabei wird zusätzlich die Gabe von Zoledronat mit der von Denosumab verglichen. Ebenfalls wird der Einfluss des RANKL-Inhibitors Denosumab in der GAIN-II-Studie geprüft. Hier wird die Infusionstherapie mit Denosumab gegenüber der oralen Einnahme von Ibandronat nach adjuvanter anthrazyklin- und taxanhaltiger Chemotherapie untersucht. 